\title{
Surgical treatment for improved 1-year survival in patients with primary cardiac sarcoma
}

\author{
Xiaowei Jiang (D), Min Yan1 (D) \\ Department of Cardiology, Xiangya Hospital, Central South University; Changsha-China \\ ${ }^{1}$ Department of Internal Medicine, Changsha Medical University; Changsha-China
}

ABSTRACT

Objective: Surgery is considered a relative contraindication in sarcoma tumor. Because of the unique characteristics of heart, whether surgery is optimally chosen in primary cardiac sarcoma (PCS) is unknown. In this study, we aimed to evaluate the 1-year survival after surgery for PCS. Methods: Patients with PCS from the Surveillance, Epidemiology, and End Results Database (SEER) between 1975 and 2015 were recruited. The endpoints were defined as 1-year all-cause mortality (ACM) and 1-year cancer-specific mortality (CSM).

Results: The study population consisted of 335 patients diagnosed with PCS. The 1-year ACM and CSM were $49.0 \%$ and $42.1 \%$ respectively. The Kaplan-Meier curves revealed that decreased 1-year ACM\&CSM were significantly associated with surgical treatment. Multiple COX regression analysis, surgery, and chemotherapy showed a significantly decreased rate of 1-year ACM and CSM. The adjusted hazard ratio of surgery was significant when the year of diagnosis was $\geq 2000$, patients were aged $<50$ years, SEER stage was localized, and patients did not undergo chemotherapy (all $p<0.05$ ), and was insignificant when the year of diagnosis was $<2000$, patients were aged $\geq 50$ years, SEER stage was distance, regional, and unstaged/unknown, and the patients underwent chemotherapy (all $p>0.05$ ). No interaction effects were detected between the variables and surgery (all $p$ for interaction $>0.05$ ).

Conclusion: Surgery should be highly recommended in patients with PCS to improve the 1-year survival rate, especially in younger patients with localized SEER stage and non-chemotherapy management.

Keywords: primary cardiac sarcoma, surgery, subgroup analysis

Cite this article as: Jiang X, Yan M. Surgical treatment for improved 1-year survival in patients with primary cardiac sarcoma. Anatol J Cardiol 2021; 25: 796-802.

\section{Introduction}

Primary cardiac tumors are extremely rare. The estimated incidence from autopsy studies ranges from $0.001 \%$ to $0.3 \%$, and less than $25 \%$ of them exhibit features of malignancy (1). Approximately, one-fourth of primary cardiac tumors are malignant with sarcomas representing the most common histology (2). Primary cardiac sarcomas (PCS) are aggressive tumors with no clinical evidence on how to manage them and continue to have a poor outcome.

Surgical treatment is usually indicated when symptoms are present and considered the mainstay of definitive therapy (3). Owing to the rarity of PCS, there is a paucity of data on the influ- ence of surgery on survival. Most research studies on PCS are case reports or literature reviews (4-7). Thus, it is necessary to carry out studies with larger patient cohorts to further assess the prognosis of PCS after surgery. The Surveillance, Epidemiology, and End Result (SEER) database is the largest cancer registry in the United States. We used the SEER database to investigate the 1 -year prognosis after surgery for patients with PCS.

\section{Methods}

\section{Study population}

The SEER ${ }^{*}$ Stat version 8.3.6 is a collection of 18 populationbased cancer registries covering approximately $34.6 \%$ of the US 


\section{HIGHLIGHTS}

- The 1-year mortality of primary cardiac sarcoma (PCS) was $49.0 \%$ in our study.

- Decreased 1-year mortality is associated with surgery and chemotherapy treatment.

- Surgery should be highly recommended in patients with PCS, especially in younger patients with localized the Surveillance, Epidemiology, and End Result stage and non-chemotherapy management.

population and was used to generate a case listing, which included PCS from 1975 to 2015. The need for informed consent was waived. The inclusion criteria were as follows: Primary Site-labeled: C38.0 (malignant neoplasm heart); Behavior code ICD-0-3: malignant. The exclusion criteria were as follows: survival time <1 month; diagnosed not from histopathology or unknown; survival months flag: incomplete data; cardiac lymphomas; other tumors (unspecified neoplasms, squamous cell carcinomas, adenocarcinomas, epithelial neoplasms, glomus tumors, and gliomas). Finally, there were 335 patients with PCS included in the study. The flow chart of patient selection is presented in Figure 1.

All the patients in the database were unidentified for privacy protection, and the need for informed consent was waived. The following information was collected for each patient: age, sex, race, size of tumor, number of tumors, classification of tumor, SEER stage, surgery, radiography, chemotherapy, and survival time. The endpoint was defined as 1-year all-cause mortality (ACM) or 1-year cancer-specific mortality (CSM).

\section{Statistical analysis}

Data analyses were performed using StataMP software version 16. Numeric variables were summarized as means (standard deviations). Categorical variables were reported as numbers (percentages). The Student test, chi-squared test, Wilcoxon rank-sum test, and Kaplan-Meier (KM) analysis were used as appropriate. The log-rank test was used to assess differences in survival experience between groups divided by surgery. Univariate and multiple Cox hazard regressions were to explore the significant factors for ACM and CSM. Subgroup analysis was performed to explore the possible interaction between surgery and mortality, and stratification was performed according to the year of diagnosis $1<2000$, $\geq 2000)$, age $(<50, \geq 50)$, SEER stage (regional, localized, distant, unstaged/unknown), and chemotherapy (yes, no). Two-sided $p$ values $<0.05$ were considered statistically significant.

\section{Results}

\section{Patient baseline characteristics}

The study population consisted of 335 patients diagnosed with PCS. Comparisons of baseline characteristics between

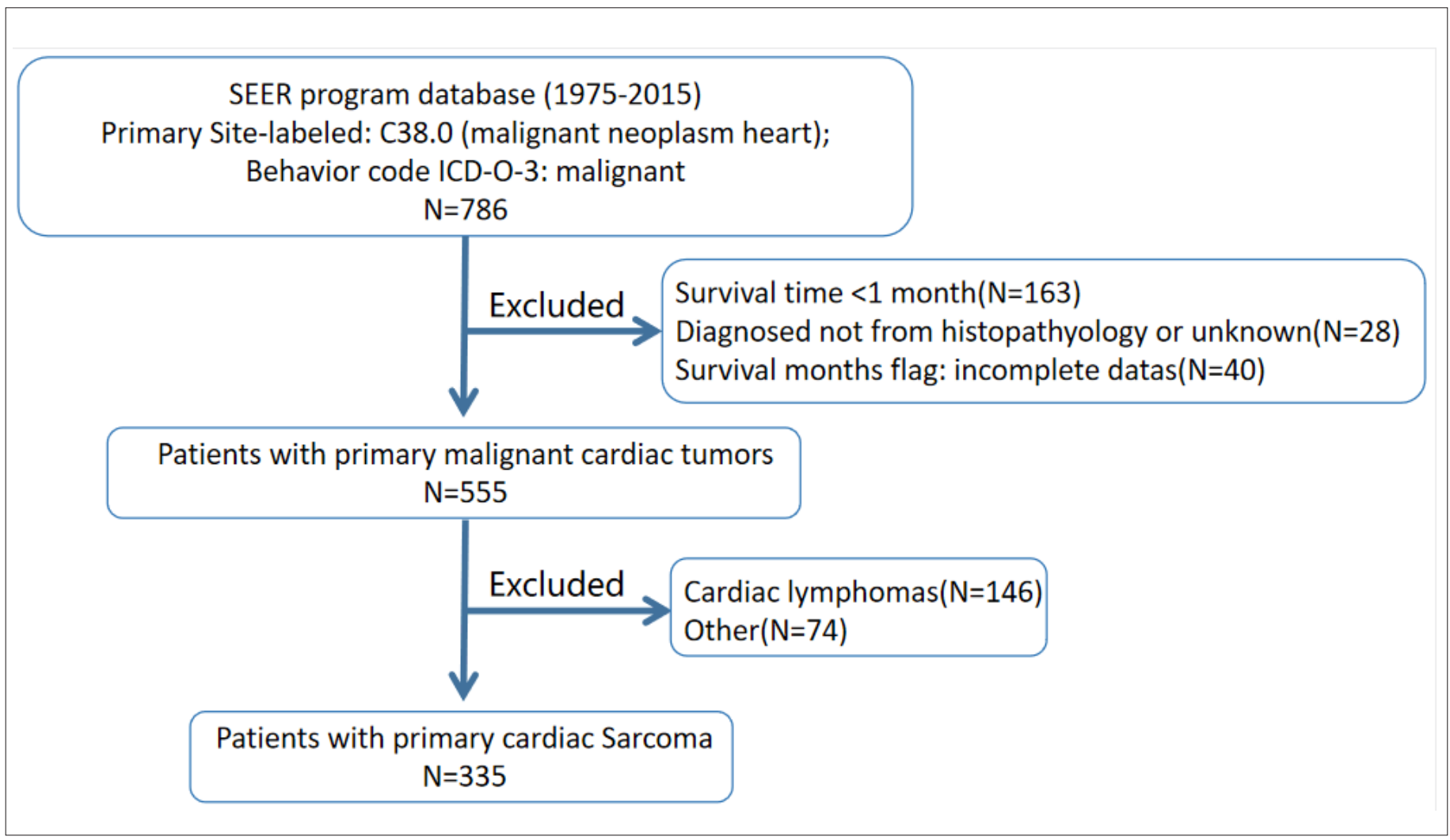

Figure 1. Flow chart of selection of study population from the SEER database

SEER - Surveillance, Epidemiology, and End Results; ICD-0-3 - International Classification of Diseases for Oncology, $3^{\text {rd }}$ edition 


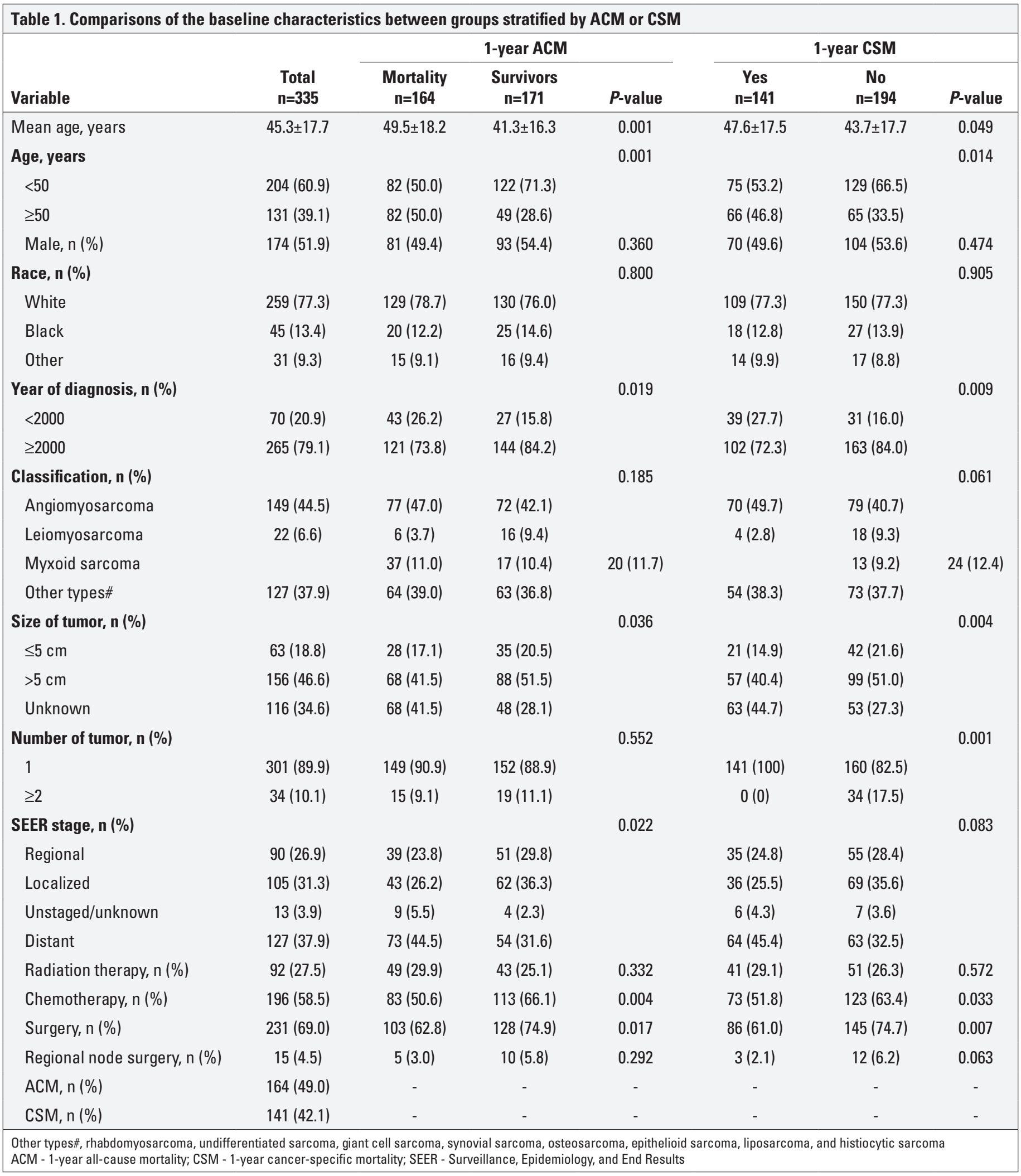

groups stratified by 1-year ACM and CSM are listed in Table 1. There were significant differences observed in age, year of diagnosis, SEER stage, size of tumor, chemotherapy, and surgery. The 1 -year ACM and CSM were $49.0 \%$ and $42.1 \%$, respectively.
Association between 1-year all-cause mortality and cancer-specific mortality and surgery

Compared with the non-surgery group, the surgery group presented significantly lower rates of 1-year ACM and CSM 
and longer survival time (Table 2). The Kaplan-Meier curves revealed that the decreased 1-year ACM and CSM were significantly associated with surgical treatment (Fig. 2). As shown in Table 3, univariate COX regression analyses showed that age, year of diagnosis, and SEER stage were significant risk factors of 1-year ACM and CSM, and chemotherapy and surgery were significant protective factors. On multiple COX regression analysis, surgical treatments and chemotherapy still showed significantly decreased rates of 1-year ACM and CSM.

Table 2. Comparisons of ACM, CSM, and survival times between the non-surgery and surgery groups

\begin{tabular}{|llll|}
\hline Variable & Non-surgery n=104 & Surgery n=231 & $\boldsymbol{P}$-value \\
\hline ACM, n (\%) & $61(58.7)$ & $103(44.6)$ & 0.017 \\
CSM, n (\%) & $55(52.9)$ & $86(37.2)$ & 0.007 \\
$\begin{array}{l}\text { Survival time, } \\
\text { months }\end{array}$ & $8(3,12)$ & $12(6,12)$ & $<0.001$ \\
\hline $\begin{array}{l}\text { Values are presented as number (\%). } \\
\text { ACM - 1-year all-cause mortality; CSM - 1-year cancer-specific mortality }\end{array}$ \\
\hline
\end{tabular}

Subgroup analysis of the association between surgery and 1-year all-cause mortality and cancer-specific mortality

Subgroup analysis was performed according to the variables revealed by Cox analysis; year of diagnosis, age, SEER stage, and chemotherapy (Fig. 3). The adjusted hazard ratio of surgery was significant when the year of diagnosis was $\geq 2000$; patients were aged $<50$ years; SEER stage was localized; and patients did not undergo chemotherapy (all $p<0.05$ ) and was insignificant when the year of diagnosis was $<2000$; patients were aged $\geq 50$ years; SEER stage was distance, regional, and unstaged/unknown; and patients underwent chemotherapy (all $p>0.05$ ). No interaction effects were detected between variables and surgery (all $p$ for interaction $>0.05$ ).

\section{Discussion}

Until now, knowledge about PCS has remained incomplete. This study evaluates the prognosis for patients with PCA diagnosed from 1975 to 2015 in the SEER registry after surgery. We found that surgery should be highly recommended to improve 1 -year survival rate in patients with PCS, especially in younger patients with localized SEER stage and with non-chemotherapy management.

\begin{tabular}{|c|c|c|c|c|c|c|}
\hline \multirow[b]{2}{*}{ Variable } & \multicolumn{3}{|c|}{ Univariable analysis } & \multicolumn{3}{|c|}{ Multiple analysis } \\
\hline & HR & $95 \% \mathrm{Cl}$ & $P$-value & HR & $95 \% \mathrm{CI}$ & $P$-value \\
\hline \multicolumn{7}{|l|}{ For ACM } \\
\hline Age $^{*}$ & 1.929 & $1.419-2.622$ & $<0.001$ & 2.083 & $1.507-2.877$ & $<0.001$ \\
\hline Year of diagnosis\# & 0.703 & $0.497-0.996$ & 0.048 & 0.632 & $0.435-0.920$ & 0.016 \\
\hline Size of tumor & 1.179 & $0.947-1.468$ & 0.141 & & & \\
\hline Number of tumors & 0.908 & $0.534-1.544$ & 0.722 & & & \\
\hline SEER stage & 0.847 & $0.711-1.000$ & 0.062 & 0.706 & $0.578-0.862$ & 0.001 \\
\hline Radiation therapy & 1.120 & $0.802-1.565$ & 0.506 & & & \\
\hline Chemotherapy & 0.593 & $0.436-0.806$ & 0.001 & 0.483 & $0.340-0.687$ & $<0.001$ \\
\hline Surgery & 0.614 & $0.447-0.845$ & 0.003 & 0.595 & $0.422-0.840$ & 0.003 \\
\hline \multicolumn{7}{|l|}{ For CSM } \\
\hline $\mathrm{Age}^{*}$ & 1.709 & $1.226-2.381$ & 0.002 & 1.898 & $1.338-2.693$ & $<0.001$ \\
\hline Year of diagnosis ${ }^{\#}$ & 0.653 & $0.451-0.944$ & 0.023 & 0.589 & $0.396-0.877$ & 0.009 \\
\hline Classification\& & - & - & 0.541 & & & \\
\hline Size of tumor & 1.319 & $1.037-1.678$ & 0.024 & & & \\
\hline Number of tumors & - & - & 1.000 & & & \\
\hline SEER stage & 0.802 & $0.663-0.969$ & 0.022 & 0.673 & $0.541-0.837$ & 0.003 \\
\hline Radiation therapy & 1.079 & $0.750-1.552$ & 0.681 & & & \\
\hline Chemotherapy & 0.619 & $0.445-0.862$ & 0.005 & 0.479 & $0.327-0.700$ & $<0.001$ \\
\hline Surgery & 0.565 & $0.403-0.794$ & 0.001 & 0.564 & $0.390-0.816$ & 0.002 \\
\hline
\end{tabular}




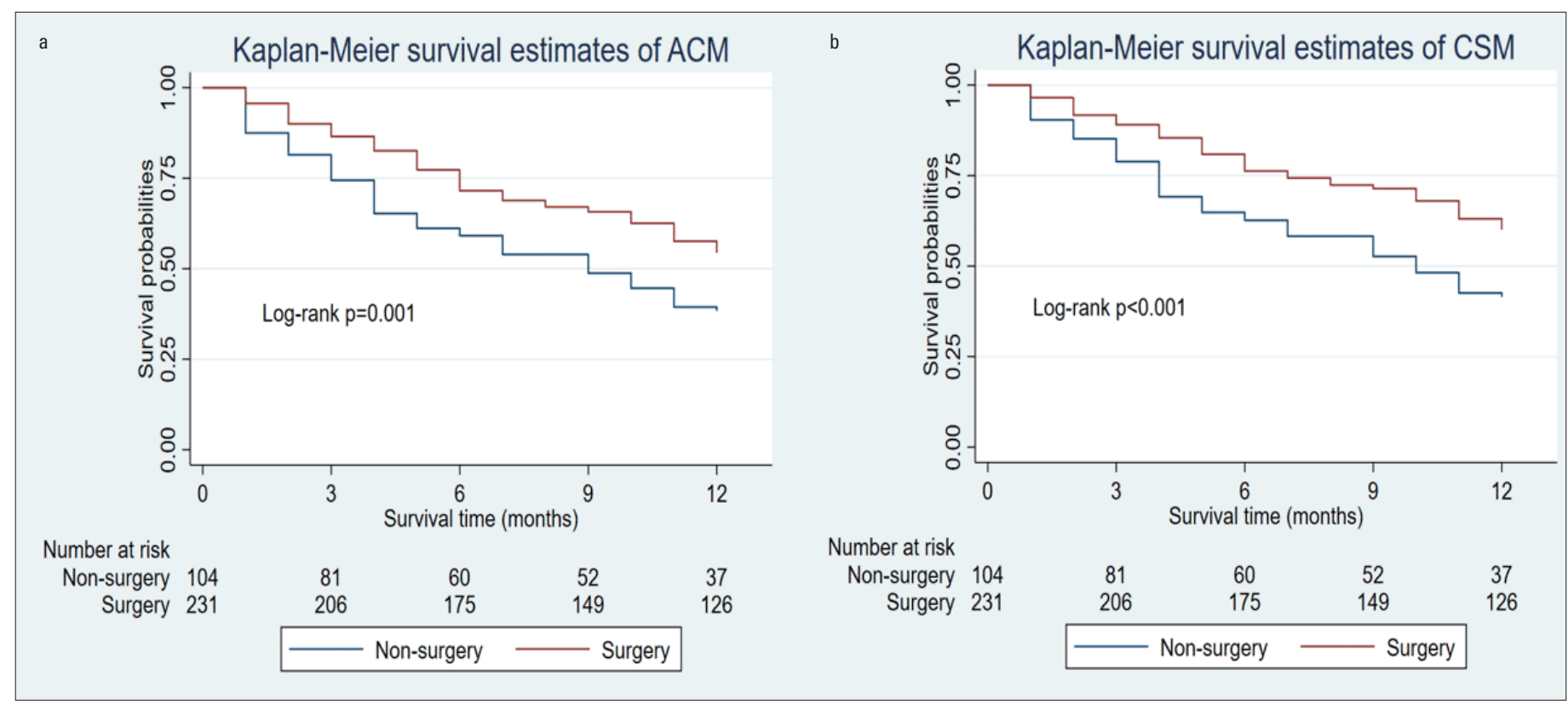

Figure 2. Kaplan-Meier method estimated 1-year ACM (a) and 1-year CSM (b) in patients with primary heart sarcoma stratified by surgery

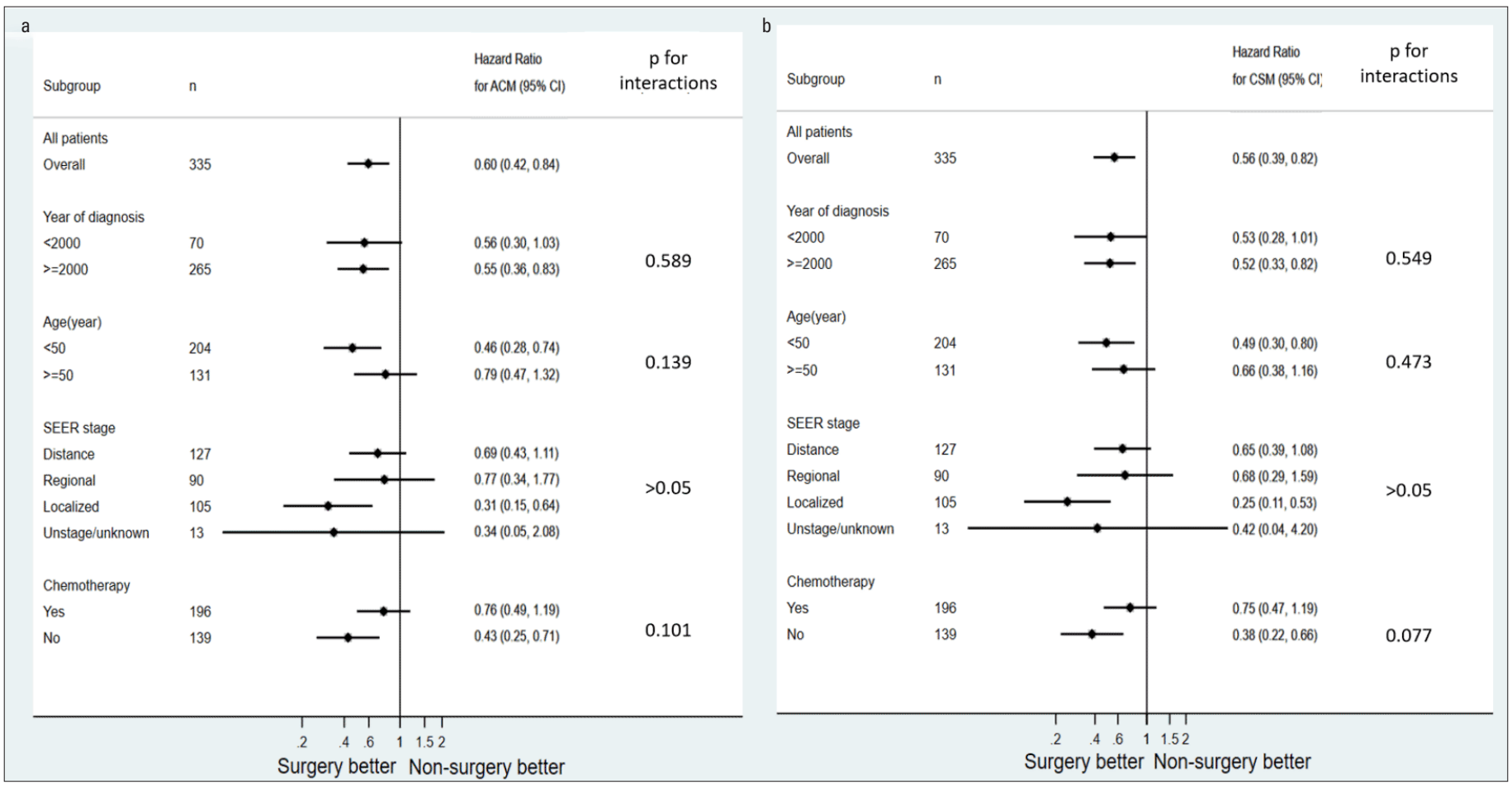

Figure 3. Subgroup analysis of the association between surgery and 1-year ACM and CSM. Using the Cox proportional hazards models, we analyzed the adjusted hazard ratios of ACM (a) and CSM (b) to surgery for the subgroup divided by the year of diagnosis, age, SEER stage, and chemotherapy SEER - Surveillance, Epidemiology, and End Results; ACM - 1-year all-cause mortality; CSM - 1-year cancer-specific mortality

The estimated incidence of primary heart tumors from autopsy studies ranges from $0.001 \%$ to $0.3 \%$ (1). Only $10 \%$ of primary heart tumors are malignant, which includes the following: Malignant teratoma; lymphoma; and various sarcomas (including rhabdomyosarcoma, angiosarcoma, undifferentiated pleomorphic sarcoma, leiomyosarcoma, chondrosarcoma, synovial sarcoma, and infantile fibrosarcoma) (8-10). Sarcomas were by far the most common malignant histology of primary cardiac tumors. Blood vessel neoplasms represent half of cardiac sarcoma cases. PCS is extremely aggressive and has a dismal prognosis, particularly for those with metastatic disease. Cardiac sarcomas have significantly worse survival compared with similar cancers of extra-cardiac origin (11). The clinical manifestations of PCS may vary and are attributed 
to the tumor location and size and also the range of tumor invasion (12).

There were significant differences observed in age, year of diagnosis, SEER stage, size of tumor, chemotherapy, and surgery between groups stratified by 1-year ACM and CSM. We used multiple Cox hazard regression analyses to explore prognosis factors, which showed that age, year of diagnosis, SEER stage, chemotherapy, and surgery had a strong prognostic association with 1 -year ACM and CSM. There is a trend of unfavorable prognosis for right- versus left-sided cardiac tumors (13). However, information about the location of tumors was not recorded in the SEER*Stat version 8.3.6 database. Angiosarcomas arise predominantly in the right atrium (14), and leiomyosarcomas arise more frequently in the left atrium (15). Left-sided cardiac sarcomas can cause heart failure early in the disease process, but they are more circumscribed, less infiltrative, have better overall survival, and metastasize later. On the contrary, right-sided cardiac sarcomas are bulky, grow in a more exophytic manner, are more infiltrative, and metastasize earlier than left-sided cardiac or pulmonary artery sarcomas. A previous study with a small sample size reported that regardless of location, attempting surgical resection remains important for a survival advantage (16).

Management of malignant cardiac tumors is challenging. Conventional treatments for malignant tumors include surgery, chemotherapy, and radiotherapy. Because of the surgical challenges presented by cardiac sarcomas and the dearth of efficacious, non-surgical therapeutic options, patients with cardiac sarcoma continue to have dismal outcomes. Chest radiography is easily accessible. However, radiography did not reach significance for mortality either in multivariate analysis or in the nomogram. As the potential benefits from radiotherapy could be balanced by long-term toxicities, radiography should be evaluated on a case-to-case basis very carefully. Chemotherapy should be highly recommended to improve prognosis in patients with PCS. Neoadjuvant chemotherapy can be used to decrease tumor mass size before surgery and enhances the surgery success ratio (17).

In our study, we found that surgery could improve the 1-year survival. Surgical resection is usually tailored on patient characteristics. Resection of PCS, even partial, is safe, provides relief from obstructive symptoms and improves quality of life (18-20). Surgery is applicable not only to clear the mechanical obstruction but also to obtain histologic material to make certain diagnoses. However, in some cases, it was concluded that aggressive surgery does not provide good results in terms of survival rate (21). When performing cardiac surgery, it should be kept in mind that the heart would have smaller and thinner walls; thus, as the preload increases following tumor resection and contractility decreases, hemodynamic complications can arise (22).

Patients selected for surgical management have more favorable survival than those selected for nonsurgical management. The association between surgery and mortality was significant in the subgroups with younger patients, localized SEER stage, and non-chemotherapy management, which suggested the feasibility of the appropriate use of surgery in these patients.
Compared with older patients, younger patients may have a relatively low risk with surgery. From the subgroup analysis of this study, it was found that surgery improved the survival of patients with PCS when they were younger than 50 years but not when they were older than 50 years. SEER stage grade was found to be an important factor associated with survival. The association between surgery and mortality was significant in the subgroup analysis only for localized SEER stage. Because surgery could not inhibit the growth and metastasis of tumor, definitive surgical therapy is not offered to all patients, and the presence of metastatic disease or concerns of limited improvement in survival remain unresolved. The chemotherapy regime for PCS was variable (23), and early high-dose systematic chemotherapy can significantly improve the prognosis (24). In patients with nonchemotherapy management for specific reasons, surgery management may be the only way to improve survival. Surgery did not show enhanced survival in patients who underwent chemotherapy in our study. A case report has shown that neoadjuvant chemotherapy can be used to decrease tumor mass size before surgery and enhance the success of surgery $(17,25)$. Thus far, no randomized trials have been conducted; and therefore, further research is needed to answer this question in the future.

\section{Study limitations}

This study has important limitations. Although the study is based on a national registry, it lacks detailed data on the location of cardiac sarcomas, dose and type of radiotherapy, chemotherapy protocol, and the indications of surgery, which limits our results and conclusion. Further prospective cohort studies are needed to provide improved insight into risk factors of survival. Cautious interpretation is required due to the retrospective nature of the study.

\section{Conclusion}

Surgery should be highly recommended in patients with primary cardiac sarcoma to improve the 1-year survival rate, especially in younger patients with localized SEER stage and nonchemotherapy management.

Conflict of interest: None declared.

Peer-review: Externally peer-reviewed.

Author contributions: Concept - X.J.; Design - X.J.; Supervision X.J.; Fundings - None; Materials - X.J.; Data collection \&/or processing - X.J.; Analysis \&/or interpretation - X.J.; Literature search - X.J., M.Y.; Writing - X.J., M.Y.; Critical review - X.J., M.Y.

\section{References}

1. Butany J, Nair V, Naseemuddin A, Nair GM, Catton C, Yau T. Cardiac tumours: diagnosis and management. Lancet Oncol 2005; 6: 219-28. [Crossref]

2. Silverman NA. Primary cardiac tumors. Ann Surg 1980; 191: 127-38. [Crossref] 
3. Blondeau P. Primary cardiac tumors--French studies of 533 cases. Thorac Cardiovasc Surg 1990; 38 Suppl 2: 192-5. [Crossref]

4. Sheppard MN, Mohiaddin R. Tumors of the heart. Future Cardiol 2010; 6: 181-93. [Crossref]

5. Burazor I, Aviel-Ronen S, Imazio M, Markel G, Grossman Y, Yosepovich $A$, et al. Primary malignancies of the heart and pericardium. Clin Cardiol 2014; 37: 582-8. [Crossref]

6. Tazelaar HD, Locke TJ, McGregor CG. Pathology of surgically excised primary cardiac tumors. Mayo Clin Proc 1992; 67: 957-65. [Crossref]

7. Bear PA, Moodie DS. Malignant primary cardiac tumors. The Cleveland Clinic experience, 1956 to 1986. Chest 1987; 92: 860-2. [Crossref]

8. Kogon B, Shehata B, Katzenstein H, Samai C, Mahle W, Maher K, et al. Primary congenital infantile fibrosarcoma of the heart: the first confirmed case. Ann Thorac Surg 2011; 91: 1276-80. [Crossref]

9. Ostrowski S, Marcinkiewicz A, Kosmider A, Jaszewski R. Sarcomas of the heart as a difficult interdisciplinary problem. Arch Med Sci 2014; 10: 135-48. [Crossref]

10. Wang JG, Li NN. Primary cardiac synovial sarcoma. Ann Thorac Surg 2013; 95: 2202-9. [Crossref]

11. Oliveira GH, Al-Kindi SG, Hoimes C, Park SJ. Characteristics and Survival of Malignant Cardiac Tumors: A 40-Year Analysis of $>500$ Patients. Circulation 2015; 132: 2395-402. [Crossref]

12. Pietras CM, Spittell PC, Nuttall GA, Eleid MF, Said SM. Pulmonary Venous Obstruction From a Large Left Atrial Sarcoma: Resection Combined With Mitral Valve Bypass. Ann Thorac Surg 2016; 102: e237-9. [Crossref]

13. Chen TW, Loong HH, Srikanthan A, Zer A, Barua R, Butany J, et al. Primary cardiac sarcomas: A multi-national retrospective review. Cancer Med 2019; 8: 104-10. [Crossref]

14. Herrmann MA, Shankerman RA, Edwards WD, Shub C, Schaff HV. Primary cardiac angiosarcoma: a clinicopathologic study of six cases. J Thorac Cardiovasc Surg 1992; 103: 655-64. [Crossref]

15. Pins MR, Ferrell MA, Madsen JC, Piubello 0, Dickersin GR, Fletcher CD. Epithelioid and spindle-celled leiomyosarcoma of the heart. Report of 2 cases and review of the literature. Arch Pathol Lab Med 1999; 123: 782-8. [Crossref]
16. Isambert N, Ray-Coquard I, Italiano A, Rios M, Kerbrat P, Gauthier $M$, et al. Primary cardiac sarcomas: a retrospective study of the French Sarcoma Group. Eur J Cancer 2014; 50: 128-36. [Crossref]

17. Blackmon SH, Patel A, Reardon MJ. Management of primary cardiac sarcomas. Expert Rev Cardiovasc Ther 2008; 6: 1217-22. [Crossref]

18. Saraiva J, Antunes PE, Carvalho L, Antunes MJ. Primary Malignant Cardiac Tumors: Surgical results. Rev Port Cardiol 2016; 35: 199204. [Article in Portuguese] [Crossref]

19. Antwi-Amoabeng D, Meghji Z, Thakkar S, Ulanja MB, Taha M, Adalja D, et al. Survival Differences in Men and Women With Primary Malignant Cardiac Tumor: An Analysis Using the Surveillance, Epidemiology and End Results (SEER) Database From 1973 to 2015. J Am Heart Assoc 2020; 9: e014846. [Crossref]

20. Saad AM, Abushouk Al, Al-Husseini MJ, Salahia S, Alrefai A, Afifi $A M$, et al. Characteristics, survival and incidence rates and trends of primary cardiac malignancies in the United States. Cardiovasc Pathol 2018; 33: 27-31. [Crossref]

21. Pacini D, Careddu L, Pantaleo A, Parolari A, Leone D, Daprati A, et al. Primary malignant tumors of the heart: Outcomes of the surgical treatment. Asian Cardiovasc Thorac Ann 2015; 23: 645-51. [Crossref]

22. Liu X, Hong H, Zhang H, Xu Z, Liu J, Qiu L. Treatment Strategies for Primary Tumors of the Heart in Children: A 10-Year Experience. Ann Thorac Surg 2015; 100: 1744-9. [Crossref]

23. Simpson L, Kumar SK, Okuno SH, Schaff HV, Porrata LF, Buckner JC, et al. Malignant primary cardiac tumors: review of a single institution experience. Cancer 2008; 112: 2440-6. [Crossref]

24. Liu S, Ren C, Wang Y. Primary Lymphoma of the Heart: A Case Report of Surgical Treatment and Review of the Literature. Heart Surg Forum 2019; 22: E225-8. [Crossref]

25. Landolsi-Ben Ammou A, Ben Fatma L, Kallel L, Gharbi O, Baâti E, Boughzela $E$, et al. Primary cardiac sarcoma: report of 3 cases and review of the literature. Ann Cardiol Angeiol (Paris) 2003; 52: 370-4. [Article in French] [Crossref] 